\title{
Generation of Primordial Cosmological Perturbations from Statistical Mechanical Models
}

\author{
A. Gabrielli, ${ }^{1}$ B. Jancovici, ${ }^{2}$ M. Joyce, ${ }^{2,3}$ J. L. Lebowitz ${ }^{4}$ L. Pietronero, ${ }^{5}$ and F. Sylos Labini ${ }^{2,5}$ \\ ${ }^{1}$ INFM UdR Roma1, Dip. di Fisica, Universitá "La Sapienza", P.le A. Moro, 2, I-00185 Roma, Italy. \\ ${ }^{2}$ Laboratoire de Physique Théorique, Université de Paris XI, Bâtiment 211, 91403 Orsay, France *. \\ ${ }^{3}$ Laboratoire de Physique Nucléaire et de Hautes Energies, Université de Paris VI, \\ 4, Place Jussieu, Tour 33 -Rez de chausée, 75252 PARIS Cedex $05^{\dagger}$. \\ ${ }^{4}$ Department of Mathematics, Rutgers University, New Brunswick, NJ08903 USA. \\ ${ }^{5}$ INFM Sezione Roma1, Dip. di Fisica, Universitá "La Sapienza", P.le A. Moro, 2, I-00185 Roma, Italy.
}

\begin{abstract}
The initial conditions describing seed fluctuations for the formation of structure in standard cosmological models, i.e. the Harrison-Zeldovich distribution, have very characteristic "superhomogeneous" properties: they are statistically translation invariant, isotropic, and the variance of the mass fluctuations in a region of volume $V$ grows slower than $V$. We discuss the geometrical construction of distributions of points in $\mathbf{R}^{3}$ with similar properties encountered in tiling and in statistical physics, e.g. the Gibbs distribution of a one-component system of charged particles in a uniform background (OCP). Modifications of the OCP can produce equilibrium correlations of the kind assumed in the cosmological context. We then describe how such systems can be used for the generation of initial conditions in gravitational $N$-body simulations.
\end{abstract}

PACS numbers: 98.80.-k, 05.70.-a, 02.50.-r, 05.40.-a

\section{INTRODUCTION}

A central problem in contemporary cosmology is the quantitative explanation of the inhomogeneity observed in the Universe at large scales (see e.g. [1, 2]). Such inhomogeneity is probed both indirectly for early times via the fluctuations in temperature in the microwave sky, and directly at present in the distribution of matter in space. All currently standard cosmological models work within a paradigm in which these fluctuations are the result of (mostly) gravitational evolution operating on some initial very small fluctuations. Common to all such models is the assumption - supported in particular by observations of the microwave background [3]- of a very specific form of these initial perturbations at large scale, i.e. Gaussian fluctuations with a Harrison-Zeldovich (HZ, often referred to as "scale-invariant") power spectrum [4, 5 . In a recent paper some of us [6] have discussed how, in a simple classification of correlated systems, this spectrum corresponds to highly ordered glass-like or lattice-like distributions. In particular such systems are characterised in real space by the striking behaviour of the variance in the mass, $\left\langle\Delta M^{2}\right\rangle$, contained in a given volume, e.g. in spheres of radius $R,\left\langle\Delta M^{2}\right\rangle \propto R^{2}$, or $R^{2} \log R$, i.e. it is essentially proportional to the surface area of the sphere. This is to be contrasted with the Poisson behaviour (proportional to the volume $R^{3}$ ) characteristic of many equilibrium systems with short range interactions away from phase transitions or that characteristic of long-range correlations at a critical point in many systems (for which $\left\langle\Delta M^{2}\right\rangle \propto R^{\alpha}$ with $\left.\alpha>3\right)$. Actually $R^{2}$ is the slowest growth possible for any isotropic translationally invariant distribution of points [7].

In this paper we examine the nature of the correlations in such super-homogeneous systems and compare them with equilibrium correlations in systems with long range interactions studied in statistical mechanics [9, 22, 23. More precisely we consider the One Component Plasma (OCP), which is simply a system of charged point particles interacting through a repulsive $1 / r$ potential, in a uniform background which gives overall charge neutrality (for a review, see [11]). We discuss how the OCP can be modified so that its equilibrium correlations are precisely those considered in cosmology. This involves an appropriately designed attractive short range potential as well as a repulsive $1 / r^{2}$ potential at large scales. The latter corresponds to a four dimensional Coulomb potential with the particles confined to three dimensions.

Our analysis of these parallels has more than theoretical interest. It also provides a new method for generating initial conditions (IC) for numerical studies of the formation of structure in cosmology [12, 13]. We explain that this method avoids the problems associated with currently used algorithms which involve superimposing small perturbations on a lattice or glass-like distribution. The latter is understood to be a "sufficiently uniform" discretization of a constant density background, not as representing the perturbed distribution. The problem with this choice of initial state is that the unperturbed distribution has its own inherent fluctuations/correlations, which can play an important role in the evolution of the system [14, 15, 16, 17].

\footnotetext{
* Unité Mixte de Recherche no. 8627-CNRS

$\dagger$ Permanent address from Sept. 1st 2002.
} 


\section{THE HZ SPECTRUM IN COSMOLOGY}

Let us recall first the necessary essentials of modeling perturbations from a uniform density used in cosmology. These are described as a continuous stationary stochastic process (SSP), with the property that the volume average of observables, i.e. empirical averages, approach the ensemble averages in the large volume limit. The stationarity refers in this context to the statistical invariance under spatial translation. Moreover one assumes also statistical isotropy of the stochastic process, i.e. invariance under rotation of the ensemble quantities. Thus if $\rho(\vec{r})$ is the density field, we have

$$
\langle\rho(\vec{r})\rangle=\rho_{0}>0
$$

and we can define a reduced 2-point correlation function $\tilde{\xi}(r)$ by

$$
\left\langle\rho\left(\vec{r}_{1}\right) \rho\left(\vec{r}_{2}\right)\right\rangle \equiv \rho_{0}^{2}[1+\tilde{\xi}(r)]
$$

where $r=\left|\vec{r}_{1}-\vec{r}_{2}\right|$. Corresponding isotropies are assumed to hold for the higher order correlation functions. Further the probability distribution for the fluctuations in the initial uniform density are generally assumed to be Gaussian.

Much more used in cosmology than $\tilde{\xi}(r)$ is the equivalent $k$-space quantity, the power spectrum $P(\vec{k})$ which is defined as

$$
P(\vec{k})=\lim _{V \rightarrow \infty} \frac{\left\langle\left|\delta_{\rho}(\vec{k})\right|^{2}\right\rangle}{V}
$$

where $\delta_{\rho}(\vec{k})=\int_{V} d^{3} r e^{-i \vec{k} \cdot \vec{r}} \delta(\vec{r})$ is the Fourier integral in the volume $V$ of the normalized fluctuation field $\delta(\vec{r})=\left(\rho(\vec{r})-\rho_{0}\right) / \rho_{0}$. In a statistically isotropic SSP this depends only on $|\vec{k}|$ with

$$
P(\vec{k}) \equiv P(k)=\int_{0}^{\infty} \tilde{\xi}(r) \frac{\sin (k r)}{k r} 4 \pi r^{2} d r .
$$

It follows from its definition that $P(k) \geq 0$, and, in the case we are considering, its integral, which is equal to $\tilde{\xi}(0)$, is assumed to be finite.

In current cosmological models it is generally assumed that the power spectrum $P(k) \propto k$ at small $k$. This is known as the HZ or "scale-invariant" power spectrum. It is believed to describe the "primordial" fluctuations at very early times, the putative remnants of a period of "inflation" prior to the ordinary Big Bang phase [1, 2]. The linear $k$ behaviour is cut-off by a short distance scale (needed to ensure integrability) i.e. for $k$ larger than some cut-off, $k_{c}, P(k)$ decreases faster than $k^{-3}$. The reason for the appearance of this particular spectrum is tied to considerations about the cosmological model (see [1, 2]). Thus in the homogeneous and isotropic Friedmann-Robertson-Walker (FRW) cosmology, the only characteristic length scale is the size of the horizon $R_{H}(t)$ (the region causally connected at a time $t$ ). The HZ spectrum then corresponds to the choice which gives (as discussed in [6] this condition is in fact only satisfied for a spherical Gaussian window function, and not in a real physical sphere)

$$
\sigma_{M}^{2}\left(R_{H}(t)\right)=\text { constant }
$$

independent of $t$,where $\sigma_{M}^{2}(R)$ is the normalized mass variance

$$
\sigma_{M}^{2}(R)=\left\langle\Delta M^{2}\right\rangle /\langle M\rangle^{2}
$$

The HZ spectrum then follows from a consistency criterion for the treatment of the perturbations in the model: any other spectrum will give mass fluctuations which dominate over the homogeneous background either at some time in the future or past.

All current cosmological models share the HZ spectrum (or something very close to it) as the "primordial" form of their perturbations. This spectrum evolves, at larger and larger scales, until the characteristic time at which the densities of matter and radiation energy are equal. This evolution which depends strongly on the particular model used may change completely the form of the spectrum for distances smaller than a characteristic scale $k_{e q}^{-1}$ (i.e. $\left.k>k_{e q}\right)$. For larger physical scales $\left(k<k_{e q}\right)$, however, the evolution is almost exactly the same in all models, and it leaves the primordial $\mathrm{HZ}$ form intact.

Viewed in the general framework of correlated processes a very crucial property of the HZ type power spectrum is that

$$
\lim _{k \rightarrow 0} P(k)=0
$$

which in real space implies that

$$
\lim _{R \rightarrow \infty} \frac{\left\langle\Delta M^{2}\right\rangle}{V(R)}=\lim _{R \rightarrow \infty} \int_{V(R)} d^{3} r \tilde{\xi}(r)=0
$$

where $V(R)$ is the volume of a sphere of radius $R$ (with arbitrary origin). Eq. (8) is to be contrasted with the behaviour of this integral in a Poisson distribution, when it yields a finite positive constant, and with that associated with power-law correlations in critical systems [6] for which the same integral of $\tilde{\xi}(r)$ diverges.

Systems satisfying Eq. (8) are thus "more homogeneous" than a Poisson type system, as can be seen when one considers the behaviour of the mass variance in spheres. For a spectrum such that $P(k) \sim k^{n}$ for $k \rightarrow 0$ and appropriately cut-off at large $k$ we have for large $R$

$$
\sigma_{M}^{2}(R) \propto\left\{\begin{array}{l}
1 / R^{3+n} \text { if } n<1 \\
\log (R) / R^{4} \text { if } n=1 \\
1 / R^{4} \text { if } n>1
\end{array}\right.
$$

In terms of the non-normalized quantity $\left\langle(\Delta M)^{2}\right\rangle \propto$ $\sigma_{M}^{2}(R) R^{6}$, Eq. (8) says that for $n>0$ we have a slower 

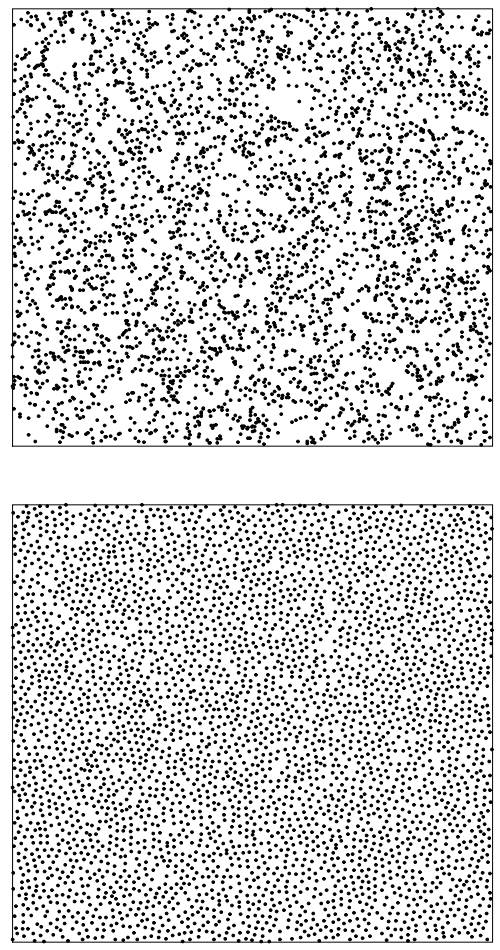

FIG. 1: A super-homogeneous distribution (bottom) and a Poisson distribution (top) with approximately the same number of points. Both are projections of thin slices of three dimensional distributions. The super-homogeneous one is a "pre-initial" configuration used in setting up the configuration representing the initial conditions for the cosmological $\mathrm{N}$-body simulations of 18 . As described in section VI the dynamics used to generate this "pre-initial" configuration gives rise to surface fluctuations as it is essentially the one component plasma which we discuss below.

increase of the variance as a function of $R$ than for Poisson fluctuations, corresponding to $n=0$, with the limiting behaviour corresponding to a variance which is proportional to the surface area of the sphere. These systems are thus characterised by surface fluctuations, ordered (or homogeneous enough, one could say) to give this very particular behaviour. In Fig. 1 are shown, in two dimensions, a Poissonian distribution and a superhomogeneous distribution. The relatively greater uniformity of the latter is clearly identifiable.

\section{DISTRIBUTIONS OF POINTS WITH SURFACE GROWTH OF THE SQUARED VARIANCE}

A simple cubic lattice is the simplest example of a discrete set of points which shows this limiting behaviour $\left(\sigma_{M}^{2} \propto 1 / R^{4}\right)$ of the variance [10]. The result is not hard to understand: The standard deviation $\sigma_{M}$ is that measured by averaging over spheres of radius $R$ centered at a randomly chosen point of the unit cell. It is proportional to the typical variation of points in the volume,

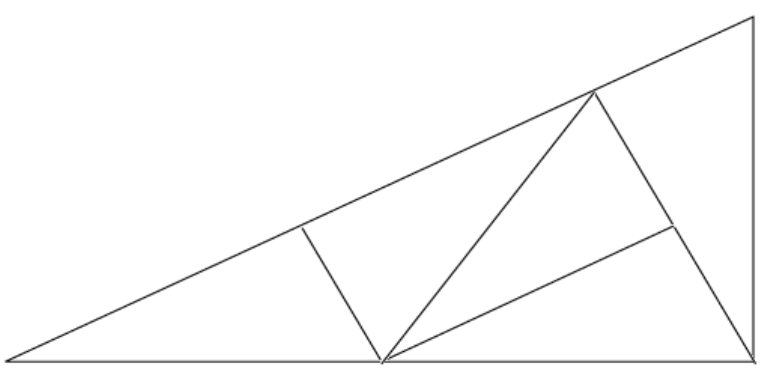

FIG. 2: Fragmentation step for the "pinwheel" tiling of the plane.)

that in the case of a lattice is the square root of the average number of points in the last spherical shell of thickness equal to the lattice unit. A better (more statistically uniform) example of the same kind is the socalled shuffled lattice [6]; this is a lattice whose sites are independently randomly displaced by a distance $x$ in all directions from their initial position according to some distribution $p(x)$ which has a finite second moment. In this case we find $P(k) \sim k^{2}$ at small $k$ and, consequently, again $\sigma_{M}^{2}(R) \propto 1 / R^{4}$ at large $R$. The simple lattice, however, is not a SSP, and even the shuffled lattice, though it can be defined as a SSP, is not statistically isotropic because the underlying lattice structure is not completely erased by the shuffling [\$].

To construct a statistically isotropic and homogeneous particle distribution with such a behaviour of $\sigma^{2}(R)$ is non-trivial. A particular example is the so-called "pinwheel" tiling of the plane [19, 20]. The generation algorithm for it is defined by taking a right angled triangle with sides of respective length one and two (and hypotenuse $\sqrt{5}$ ) and, at the first step, forming five similar square triangle of sides $1 / \sqrt{5}$ and $2 / \sqrt{5}$ respectively as shown in Fig. 2. At the second step we expand these new triangles, to the size of the original triangle, and repeat the procedure ad infinitum, so that they cover the plane completely. Finally, placing a point randomly inside each elementary triangle will give the superhomogeneous point distribution which is statistically isotropic (with a continuous power spectrum).

\section{A PHYSICAL EXAMPLE: THE ONE-COMPONENT PLASMA}

In the study of the one component plasma (OCP) one considers the equilibrium statistical mechanics (at inverse temperature $\beta$ ) of a system of charged point particles interacting through a Coulomb potential, in a continuous uniform background giving overall charge neutrality [11. Taking the particles to carry unit mass and charge the 
microscopic mass density of the particles is given by

$$
\rho(\vec{r})=\sum_{i} \delta\left(\vec{r}-\vec{r}_{i}\right)
$$

where $\vec{r}_{i}$ is the position of the $i$ th particle. The total microscopic charge density is

$$
\rho_{c}(\vec{r})=\sum_{i} \delta\left(\vec{r}-\vec{r}_{i}\right)-n
$$

where

$$
n=\langle\rho(\vec{r})\rangle .
$$

The two-point correlation function is defined as

$$
\begin{aligned}
\tilde{\xi}\left(\vec{r}-\vec{r}^{\prime}\right) & =\frac{\left\langle\rho(\vec{r}) \rho\left(\vec{r}^{\prime}\right)\right\rangle}{\langle\rho\rangle^{2}}-1 \\
& =\frac{\delta\left(\vec{r}-\vec{r}^{\prime}\right)}{n}+h\left(\vec{r}-\vec{r}^{\prime}\right) .
\end{aligned}
$$

The first term comes from the diagonal terms $\vec{r}=\vec{r}^{\prime}$ in $\left\langle\rho(\vec{r}) \rho\left(\vec{r}^{\prime}\right)\right\rangle$ and is characteristic of any point distribution.

It can be proven quite generally [9] that at high temperatures the OCP has a translation invariant isotropic distribution with $\tilde{\xi}(\vec{r})$ satisfying Eq. (8) with $r=|\vec{r}|$. In terms of $h(r)$ this gives

$$
n \int h(r) d^{3} \vec{r}=-1
$$

Eq.(14) is usually referred to as a sum rule which originates from the perfect screening of each charge caused by the long-range nature of the Coulomb potential. To see this let us suppose that an external infinitesimal charge density $\rho_{\text {ext }}=\epsilon e^{i \vec{k} \cdot \vec{r}}$ of very long wavelength is applied to the system. It creates an external electric potential

$$
\Phi(\vec{r})=\int \frac{\rho_{\mathrm{ext}}\left(\vec{r}^{\prime}\right)}{\left|\vec{r}-\vec{r}^{\prime}\right|} d^{3} \vec{r}^{\prime}=\frac{4 \pi}{k^{2}} \epsilon e^{i \vec{k} \cdot \vec{r}}
$$

and a perturbation to the Hamiltonian

$$
H_{\mathrm{ext}}=\int \rho_{c}(\vec{r}) \Phi(\vec{r}) d^{3} \vec{r}=\epsilon \frac{4 \pi}{k^{2}} \int \rho_{c}(\vec{r}) e^{i \vec{k} \cdot \vec{r}} d^{3} \vec{r} .
$$

Using linear response theory the induced charge in the system is given by

$$
\rho_{\text {ind }}\left(\vec{r}^{\prime}\right)=-\beta\left\langle\rho_{c}\left(\vec{r}^{\prime}\right) H_{\text {ext }}\right\rangle
$$

where the average is over the unperturbed statistical distribution. Thus, assuming that the applied charge is perfectly screened i.e. the system responds with an induced charge density $\rho_{\text {ind }}=-\rho_{\text {ext }}$ we have, in the limit, $k \rightarrow 0$,

$$
-\epsilon e^{i \vec{k} \cdot \vec{r}^{\prime}} \sim-\beta \epsilon \frac{4 \pi}{k^{2}} \int\left\langle\rho_{c}\left(\vec{r}^{\prime}\right) \rho_{c}(\vec{r})\right\rangle e^{i \vec{k} \cdot \vec{r}} d^{3} \vec{r}
$$

and therefore, since

$$
P(k)=\frac{1}{n}+\tilde{h}(k)
$$

where $\tilde{h}(k)$ is the Fourier transform of $h$, we find that

$$
P(k) \sim \frac{k^{2}}{4 \pi n^{2} \beta}
$$

for small $k$.

The behaviour of the power spectrum at small $k$ is traceable through Eq. (15) as being simply, up to a factor $\beta$, the inverse of the Fourier transform, (in the sense of distributions) of the repulsive $1 / r$ potential. It is evident that what one needs to obtain the same kind of behaviour but with $P(k) \sim k$ at small $k$ is a repulsive potential of which the Fourier transform behaves as $1 / k$, i.e. a repulsive $1 / r^{2}$ potential. Before considering this in more detail we discuss some issues relevant to the cosmological context which we have passed over without comment so far.

\section{DISCRETE VS. CONTINUOUS}

An essential difference between the case of cosmological perturbations and the system just discussed is that the former refers to a continuous density field while the latter describes the correlation properties of a set of discrete points. To interpret the latter as giving a realization of the former we obviously need to specify explicitly how to relate the two. There is no unique prescription to pass from a discrete to a continuous field. A prescription simply corresponds to a regularisation of the Dirac delta with a function $W_{L}(\vec{r})$ with the property

$$
W_{L}(\vec{r})=L^{-3} W_{o}\left(\frac{\vec{r}}{L}\right), \quad \int W_{o}(\vec{r}) d^{3} \vec{r}=1
$$

where $L$ is the characteristic scale introduced by the regularisation e.g. the Gaussian

$$
W_{L}(\vec{r})=\left(\frac{1}{\sqrt{2 \pi} L}\right)^{3} \exp \left(-\frac{r^{2}}{2 L^{2}}\right) .
$$

For any finite value of $L$ we can define a continuous density field $\rho_{L}(\vec{r})$ as the convolution of this function with the discrete density field. The pair correlation function of the continuous field can then be written also as a double convolution integral of $W_{L}(\vec{r})$ and the correlation function in the discrete case. The singularity in the latter is thus also removed by the regularisation. The power spectrum of this continuous field is then simply given as

$$
P_{L}(\vec{k})=\left|W_{L}(\vec{k})\right|^{2} P_{D}(\vec{k})
$$

where $W_{L}(\vec{k})$ is the Fourier transform of the regularisation $W_{L}(\vec{r})$ and $P_{D}(\vec{k})$ is the power spectrum of the discrete field. In particular for the Gaussian smoothing function we have

$$
P_{L}(\vec{k})=\exp \left(-k^{2} L^{2}\right) P_{D}(\vec{k}) .
$$


For the OCP discussed above the correlation function, in the weak coupling limit, is approximately the DebyeHückel formula

$$
h(r)=-\beta \frac{e^{-\kappa r}}{r} \quad \kappa^{2}=4 \pi \beta n
$$

so that

$$
P_{D}(\vec{k})=\frac{1}{n}\left[\frac{k^{2}}{\kappa^{2}+k^{2}}\right]
$$

which is a non-integrable function of $k$. Integrability is ensured by the cut-off imposed at large $k$ in the power spectrum through the smoothing function. An assumed property of the primordial density field in standard cosmological models is that it is Gaussian distributed. Since the density field is inherently positive this assumption of Gaussianity is more properly attributed to fluctuations (expected to be small) around the uniform density. Small fluctuations in the discrete OCP are in fact Gaussian [22.

\section{INITIAL CONDITIONS IN N-BODY SIMULATIONS}

A context in which it is necessary to have a concrete realization of the density $\rho(\vec{r})$ in cosmological models is that of the N-body approach to the problem of structure formation 12, 13. In these numerical simulations an initial configuration (IC), which should represent the universe at early times, is evolved under its self-gravity. The goal of these simulations is an understanding of how structures grow in such models, and whether they are compatible with observations of the distribution of matter (as probed, primarily, by the distribution of galaxies and galaxy clusters). The initial continuous density field is thus necessarily represented by a discrete set of points, which, because the discretization scale does not represent the real underlying physical one, must be considered just as we have discussed above as representing the continuous density field via some appropriate smoothing.

In practice such a discretization is always generated in a very particular way [13, 24]: points of equal mass are displaced from a "pre-initial" configuration in a way prescribed by the correlation field one wants to set up. This prescription can be understood as follows. Superimposing an infinitesimal displacement field $\vec{u}(\vec{r})$ on a uniform density $\rho_{o}$ one has

$$
\frac{\rho(\vec{r})-\rho_{o}}{\rho_{o}} \simeq-\vec{\nabla} \cdot \vec{u}(\vec{r})
$$

and therefore in $k$-space the power spectrum of the correlated density field $\rho(\vec{r})$ produced is roughly proportional to $k^{2} P_{u}(k)$, where $P_{u}(k)$ is that of the displacement field. The "pre-initial" configuration used, which gives the initial unperturbed positions of the particles, is a discretization of the initial uniform density field $\rho_{o}$ and the displacement field is specified by the power spectrum of the continuous field which is desired as IC in the simulation. In practice the former is taken either to be an exact simple lattice or a "glassy" configuration [13. When this latter configuration is used it is generated by evolving the system with the N-body code, but with the sign of gravity reversed. This corresponds to a system essentially like the OCP (at low temperature), and the long-time evolution brings it to a highly uniform configuration with normalized variance decaying at large scales as $1 / R^{4} 15$.

In the generation of the IC in this way the fluctuations associated with the "pre-initial" configuration are neglected, or rather implicitly assumed to be negligible with respect to those which are introduced by the perturbations to it. The problem with this procedure is that it neglects precisely these "pre-initial" fluctuations. (If we assume the initial continuous field to have powerspectrum $P_{i}(k)$ what results from the infinitesimal shuffling is a power spectrum $\left.P(k)=P_{i}(k)+k^{2} P_{u}(k)\right)$. Such a neglect is justified if these fluctuations play no role in the evolution of the system, as one wants to see only the growth of structure coming from the perturbations superimposed on the uniform density field. Indeed the reason the lattice or "glassy" configuration is used is that they are configurations in which the gravitational force is (effectively) zero, whereas in a Poisson distribution for example (which is a priori as good a discretization of the uniform background) it is not. While the Poisson distribution will thus evolve and form structures even without additional perturbation, the perfect lattice will not. That the discretized lattice or glass solves this problem is however far from clear: they both represent unstable point configurations with respect to gravity. A small applied perturbation will thus in general give an evolution which depends on the correlations and/or fluctuations in the initial unperturbed configurations. In principle such effects can be kept under control by making the discretization of the original density sufficiently fine. In practice in N-body simulations the "non-linear regime" in which structure formation takes place is comparable to the discretization scale, and the role of such effects seems, at the very least, problematic [14, 15, 16, 17].

One way in which this difficulty could be got around is by generating directly a discrete distribution whose regularisation is exactly the desired continuous field. Our observations on the OCP provides in principle a way of realizing this possibility.

\section{IC AS EQUILIBRIUM OF A MODIFIED OCP}

We have seen that the OCP equilibrium correlations give surface fluctuations $\left(\left\langle\Delta M^{2}\right\rangle \sim R^{2}\right)$, but with a power spectrum at small $k$ which goes like $k^{2}$. By considering instead a repulsive $1 / r^{2}$ potential, whose Fourier transform is $2 \pi^{2} / k$, we obtain from Eq. (19)

$$
P(k) \sim \frac{k}{2 \pi^{2} n^{2} \beta}, \text { for } k \sim 0
$$


and

$$
h(r) \sim-\frac{1}{2 \pi^{4} \beta n^{2}} \frac{1}{r^{4}}
$$

for $r \gg\left(2 \pi^{2} \beta m\right)^{-1}$. The change from the exponential decay of Eq. (25) to a power-law decay is a result of the different analyticity properties of the two power-spectra: the $k^{2}$ behaviour is analytic at the origin, guaranteeing a rapidly decaying behaviour of its Fourier transform, while the $k$ spectrum is not.

In the context of cosmological N-body simulations what one needs is not simply the primordial HZ power spectrum with some appropriate small scale cut-off: what is simulated is only a part of the cosmological evolution, starting from a time at which the initial spectrum of fluctuations is already significantly modified from its primordial form. While purely gravitational evolution at these early times does not modify the HZ spectrum, nongravitational effects, present until the time when the universe becomes dominated by matter, do so significantly. The nature of these modifications depends on the details of the cosmological model, but in all cases it affects only $k$ larger than a characteristic $k_{e q}$ corresponding to the causal horizon at the "time of equality" (when matter starts to dominate over radiation). One then has a power spectrum of the form

$$
P(k)=k^{n} f(k)
$$

with $n$ exactly (or very close to) unity and $f(k)$ a modeldependent form for the spectrum with $f(0)=a>0$. In Figure 3 are shown the power spectra of two representative cosmological models [1, 2]. Both are CDM ("cold dark matter") type. HDM ("hot dark matter") models, now strongly disfavored, have a more abrupt (typically exponential) cut-off at larger $k$, due to the "wash-out" of small scale structure associated with their higher velocity dispersions.

To produce such a spectrum as the equilibrium one of an OCP like system requires further modification of the form of the potential. Just like the standard OCP, an unmodified $1 / r^{2}$ potential will give, in the weak-coupling limit, a spectrum which becomes flat (i.e. Poisson like) at large $k$. This is simply due to the fact that in this limit the thermal fluctuations dominate over the potential at small scales, effectively randomizing the particle positions up to some scale. A crude guess of what potential would produce the behaviour of a typical cosmological model can be obtained by supposing that at small scales

$$
1+h(r) \approx e^{-\beta V(r)}
$$

which corresponds to completely neglecting collective effects $(1+h$ represents the relative probability compared to random of finding a particle at distance $r$ from a given one). Given that the desired fluctuations always have small amplitudes $(|h| \ll 1)$, we would then need to be in the regime of temperature and scales such that

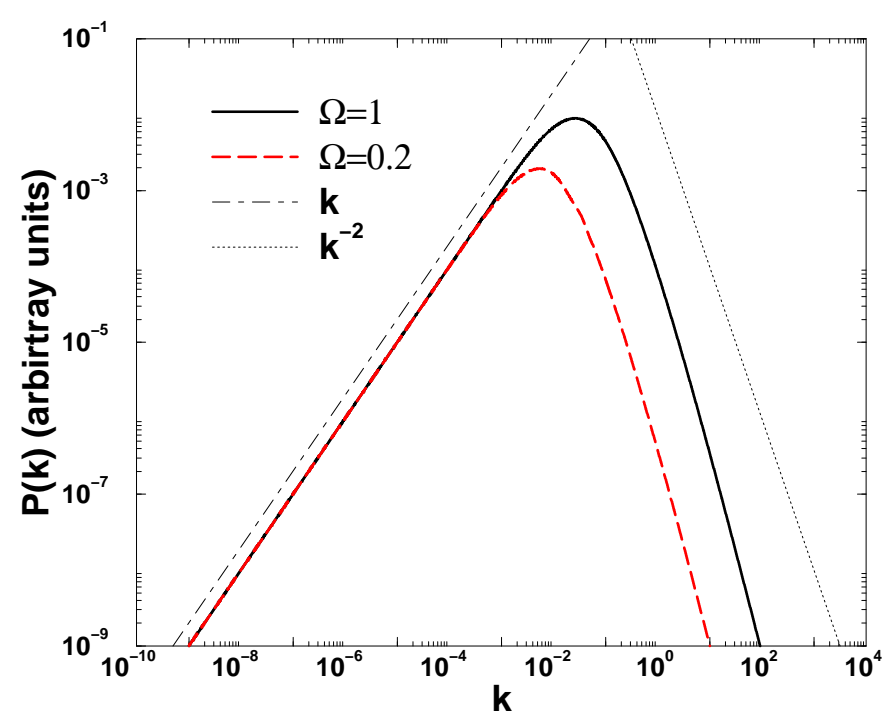

FIG. 3: Power spectra of two different cosmological models, both of the CDM ("cold dark matter") type but with a differing density of matter (parametrised by $\Omega$ ). The plot is in $\log$ - $\log$ units so that the behaviour $P(k) \sim k$ and $P(k) \sim k^{-2}$ correspond to straight lines of slope +1 and -2 respectively.

$|\beta V(r)| \ll 1$, so that $h(r) \sim-\beta V(r)$. The potential should thus be attractive at smaller scales, as the system is positively correlated at those scales. $\mathrm{A} k^{-2}$ behaviour at large $k$, which is often used (see Figure 3) as an initial condition approximating cosmological models (CDM type) in this regime (beyond the "turn-over"), may be obtained from an attractive $1 / r$ potential, e.g.

$$
V(r)=\frac{1}{r^{2}}-\eta \frac{e^{-\mu r}}{r} .
$$

By modifying the parameters $\mu$ and $\eta$, as well as the temperature, both the amplitude of the $P(k)$ and the location of a change from a $P(k) \sim k^{-2}$ to a behaviour $P(k) \sim k$ for small $k$ can be controlled. The potential (32) is repulsive at short distances, but it may be necessary to make it more strongly repulsive in order to to ensure the system is not unstable to collapse 25]. Such issues, as well as the practical numerical implementation of these observations will be investigated in forthcoming work. Once this method has been implemented to set up the initial conditions, it will naturally be interesting to understand the effect of this change in initial conditions on the dynamical evolution of gravitational N-body simulations.

\section{ACKNOWLEDGMENTS}

We thank Dominique Levesque for useful discussions about the OCP and Charles Radin for information about 
pinwheel tilings. FSL thanks the Swiss National Science Foundation for post-doctoral support. The work of JLL was supported by NSF grant DMR 98-13268 and AFOSR grant AF 49620-01-1-0154.

[1] P. J. E. Peebles, Principles of physical cosmology (Princeton University Press, NJ, 1993)

[2] T. Padmanabhan, Structure formation in the universe (Cambridge University Press, Cambridge, England, 1993).

[3] C.L. Bennett et al., Astrophys.J., 436, 423 (1994).

[4] E.R Harrison, Phys.Rev. D 1, 2726 (1970).

[5] Ya.B. Zeldovich, Mon.Not.R.Acad.Soc. 160, 1 (1972).

[6] A. Gabrielli, M. Joyce and F. Sylos Labini, Phys.Rev. D65, 083523 (2002)

[7] J. Beck, Acta Mathematica 159, 1-878282 (1987).

[8] P. Gács and D. Szász, Annals of Probability 3, 597(1975).

[9] Ph. A. Martin, Rev. Mod. Phys. 601075 (1988).

[10] D. G. Kendall and R. A. Rankin, Quart. J. Math. Oxford (2) 4, 178 (1953).

[11] M. Baus and J.-P. Hansen, Physics Reports 59, 1 (1980).

[12] R.W. Hockney and J.W. Eastwood, Computer simulation using particles (McGraw-Hill, New York, 1981).

[13] S.D.M. White, Lectures given at Les Houches astro-ph/9410043 (1993).

[14] R.J. Splinter, A.L. Melott, S.F. Shandarin and Y. Suto, Astrophys.J. 497, 38 (1998).

[15] T. Baertschiger and F. Sylos Labini, Europhys Lett., 57, 322 (2002).

[16] T. Baertschiger, M. Joyce and F. Sylos Labini, astro-ph/0203087 (2002).

[17] F. Sylos Labini, T. Baertschiger and M. Joyce, astro-ph/0207029 (2002).

[18] A. Jenkins et al., Astrophys.J., 499, 20 (1998).

[19] C. Radin, chapter in Geometry at Work, MAA Notes, 53 (Math. Assoc. Amar., Washington, DC, 2000).

[20] C. Radin, Notices Amer. Math. Soc., 42, 26 (1995).

[21] B. Grünbaum and G. C. Shepard, Tilings and patterns (Freeman, New york, 1986).

[22] Ph.A. Martin and T. Yalcin, J. Stat. Phys., 22, 435 (1980).

[23] D. Levesque, J-J. Weis and J. Lebowitz, Journal of Stat. Phys., 100, 209 (2000).

[24] G. Efstathiou, M. Davis, C. Frenk and S. White, Astrophys.J. Supp. 57, 241 (1985).

[25] D. Ruelle, Statistical Mechanics (Benjamin, New York, 1969). 